\title{
Memory for actions in scripted activities as a function of typicality, retention interval, and retrieval task
}

\author{
DONALD A. SMITH and ARTHUR C. GRAESSER \\ California State University, Fullerton, California 92634
}

\begin{abstract}
This study examined memory for information that varies in typicality (relevance) to a central organizing schema. Subjects listened to scripted activities and were given recall and recognition tests after different retention intervals. Data supported a schema-pointer-plus-tag model that specifies how memory for scripted actions varies as a function of typicality, retention interval, and retrieval task. Several mathematical formulations of the model were tested in order to simulate the recall and recognition data. The model that best fit the data incorporated four properties. First, memory discrimination is initially better for atypical than for typical actions in both recall and recognition tasks. Second, recall involves conceptually driven retrieval, whereas recognition involves both conceptually driven and data-driven retrieval. Third, conceptually driven retrieval has an exponentially decreasing retention function, whereas there is a linear decrease in data-driven retrieval. Fourth, the retention function for conceptually driven retrieval is steeper for atypical than for typical actions, which supports the notion that the schema plays a more important role in guiding this type of retrieval as the retention interval increases; in contrast, the slopes of the retention functions for data-driven retrieval are the same for typical and atypical actions. A schema-pointer-plus-tag model was compared with some alternative schema-based memory models.
\end{abstract}

In recent years, there has been increasing interest in developing models to explain the role of schemata in the acquisition, representation, and retrieval of information in memory (Bower, Black, \& Turner, 1979; Cantor \& Mischel, 1979; Graesser, Woll, Kowalski, \& Smith, 1980; Hastie, 1980; Kintsch \& van Dijk, 1978; Norman \& Bobrow, 1979; Rumelhart \& Ortony, 1977; Spiro, 1977; Taylor \& Crocker, 1981; Thorndyke \& HayesRoth, 1979). With regard to prose comprehension, most researchers agree that schemata are generic knowledge structures that interrelate statements in passages. For example, when individuals comprehend a passage about someone eating in a restaurant, they identify the generic restaurant schema. This schema provides a knowledge base that guides the interpretation of the text and generates inferences and expectations.

With regard to the representation and retrieval of information in prose, there are several issues that a schema-based model might address. One issue pertains to the typicality of information with respect to a central organizing schema. It is rare that specific experiences are perfectly consistent with the generic schemata that

This research was supported by Grant MH-33491 from the National Institute of Mental Health. We would like to thank John Black for his insightful comments on this manuscript. We would also like to thank Martin McMullen for his technical assistance on the figures and Springer-Verlag for granting permission to use Figure 1 and Table 1. Requests for reprints should be sent to Arthur C. Graesser, Department of Psychology, California State University, Fullerton, California 92634. organize them. There are usually aspects of an experience that deviate from an established schema. Information may be irrelevant to an identified schema or even inconsistent with the schema. Consider the following short passage.

"Jack was hungry so he went to a restaurant. He walked in, was seated by the hostess, and ordered food. After he finished his meal, Jack sipped his coffee and cleaned his glasses. The waitress brought Jack his bill. He left a tip, paid the bill, and left the restaurant."

Most of the statements in this passage are relevant to a restaurant schema, whereas "cleaning glasses" is clearly irrelevant. Specific information varies in typicality (or relevance) with respect to the generic schema. A schema-based model should explain how memory is affected by variations in typicality.

A second issue for schema-based models involves retrieval processes in recall and recognition. In previous years, there has been considerable debate over the similarities and differences between recall and recognition processes (Brown, 1976; Kintsch, 1977; G. Mandler, 1972, 1980; Rabinowitz, G. Mandler, \& Patterson, 1977; Tulving \& Thomson, 1973). It is clear that recall involves organized strategies of retrieval, but there has been some debate about the role of organizational variables in recognition memory. For example, Graesser et al. (1980) proposed that the organized retrieval processes that operate at recall are executed to some extent at recognition (see also Atkinson \& Juola, 1974; G. Mandler, 1972; G. Mandler \& Boeck, 1974; 
Rabinowitz et al., 1977). However, "copy cues" at recognition frequently invoke additional retrieval processes or decision processes (Atkinson \& Juola, 1974). that do not operate during recall. A complete schema model would specify mechanisms underlying both recall and recognition.

A third issue for schema-based models is the effect of retention interval on the retrieval of information from memory. Schema models have often specified how retention interval influences the retrieval of information, but the various models do not offer the same predictions. Specifically, some schema models predict equal decay rates for typical and atypical information (Cohen, 1981; Hastie, 1980). Other models predict different decay rates for atypical and typical information (D'Andrade, 1974; Graesser et al., 1980; J. Mandler, 1979; Wyer \& Srull, 1980). If the schema plays a more important role in guiding retrieval as the retention interval increases, then there would be a faster decay rate for atypical than for typical information. For the present purposes, we use the term "decay" in a quantitative sense. The inability to retrieve information is likely to be the product of some type of interference.

A fourth issue addresses the guessing that occurs on both recall and recognition tests. It is a common procedure to correct for guessing on recognition tests. It is also necessary to include a correction for guessing on recall protocols. When individuals recall passages, they often recall typical information that is not presented in the acquisition passage. For example, if an individual read a passage about someone going to a restaurant, the individual would most likely recall that the person ate, even if the action of eating was not specifically stated in the text. A similar bias in recognition tests results in a high rate of false recognitions for typical actions (Bower et al., 1979; Graesser, Gordon, \& Sawyer, 1979; Graesser et al., 1980). In both types of tests, it is important to use a measure of memory discrimination that corrects for guessing biases.

The present study was designed to test some basic assumptions and predictions of a schema-pointer-plustag (SP+T) model, with a specific focus on the four issues discussed above. The SP+T model has been examined in some detail in recent studies (Graesser, 1981; Graesser et al., 1979, 1980). The model has properties that are similar to the "schema-with-correction" hypothesis introduced decades ago (Bartlett, 1932; Woodworth, 1958; Woodworth \& Schlosberg, 1954).

The $\mathrm{SP}+\mathrm{T}$ model is based on certain representational and retrieval assumptions. As shown in Figure 1, the model assumes that the memory representation of a schema-based passage consists of (1) a pointer to the generic schema that interrelates both the stated and inferred very typical actions as a whole, (2) a set of tags that link moderately typical items to the generic schema, and (3) a set of tags that link atypical items to the memory representation. The fact that the pointer links

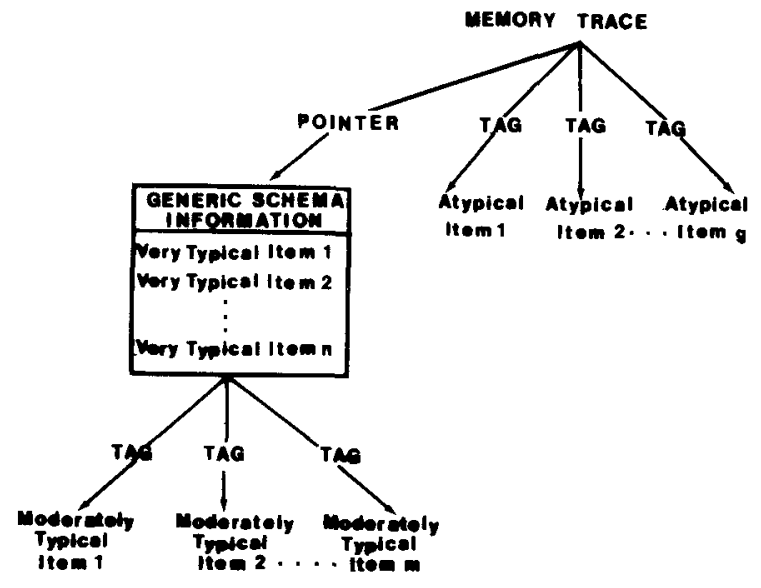

Figure 1. Representation of a schema-based passage according to the schema-pointer-plus-tag model. (From Prose Comprehension Beyond the Word by A. C. Graesser. Copyright 1981 by Springer-Verlag New York, Inc. Reprinted by permission.)

the memory trace to the schema as a whole implies that the schema is accessed in an all-or-none manner and that most or all of the schema-relevant information is copied into the specific memory trace that is constructed for a specific passage or excerpt. Therefore, a substantial portion of the unstated typical information is filled in by default (Minsky, 1975; Norman \& Bobrow, 1979). At retrieval, the individual has some likelihood of recovering any given tagged atypical item or tagged marginally typical item. There is some likelihood of guessing that a nontarget typical item was presented, because such an item would be inferred at comprehension or at retrieval.

Two studies have supported the SP+T model's predictions regarding memory for actions as a function of typicality (Graesser et al., 1979, 1980). In these studies, there was better memory discrimination for atypical than for typical actions and no memory discrimination for very typical actions. Both of these studies used scripted passages as stimulus materials. Scripts are schemas that correspond to conventional or frequently enacted activities (e.g., eating in a restaurant or washing a car; see Schank \& Abelson, 1977).

With regard to retrieval, the $\mathrm{SP}+\mathrm{T}$ model postulates two distinct processes that determine a subject's correct retrieval of information that had been presented in the acquisition material. The first process will be called conceptually driven retrieval. In conceptually driven retrieval, organized retrieval strategies access a specific item that is part of a contextually specific memory trace. Conceptually driven retrieval strategies are structured and implemented in an organized manner; the relevant generic schema plays a central role in guiding this organized retrieval. The context in which an item is embedded is also important. In conceptually driven retrieval, an item would not be retrieved without its context being reinstated. Recall is guided exclusively by conceptually driven retrieval. 
The second component of the proposed retrieval mechanism will be called data-driven retrieval. The test item on the recognition test contains a number of informational cues that provide relatively direct access to the item in memory. Data-driven retrieval is directed by the item itself as a "copy cue" (Rabinowitz et al., 1977). Data-driven retrieval is analogous to what has been called "detection of familiarity" (Atkinson \& Juola, 1974) or "intraitem elaboration" (G. Mandler, 1980) in some models of recognition memory. Data-driven retrieval is not always guided by organized retrieval strategies and does not always critically depend on reinstating the organized context in which a particular item is embedded. For example, an atypical action may be correctly recognized without the subject's remembering in which scripted activity the action occurred. Recognition mechanisms are determined by both conceptually driven and data-driven retrieval.

In order to test the effects of varying retention intervals on the retrieval of script actions, Graesser et al. (1980) collected both recall and recognition data after 30-min and 1-week retention intervals. Two trends emerged from the data. First, retention was initially better for atypical actions than for typical actions. This finding occurred for both recall and recognition tests. Second, atypical actions were forgotten at a faster rate than typical actions were for both recall and recognition. The differential forgetting rates were obvious when examining a crossover pattern in the recall data. Whereas recall was better for atypical than for typical actions after a 30-min retention interval, recall was better for typical than for atypical actions after a 1-week retention interval. Graesser et al. (1980) concluded that the generic schema plays an increasingly more important role in guiding conceptually driven retrieval (recall) as the retention interval increases. However, the question of whether or not a schema has similar effects on data-driven retrieval over time is still unanswered. Since the previous study included only two data points, it was necessary to extend the retention intervals in order to discover the precise nature of both recall and recognition decay functions.

There were three major goals in the present study. The first goal was to assess the extent to which the SP+T model explains how memory for actions in scripted activities is influenced by typicality, retention interval, and retrieval task (recall vs. recognition). The second goal was to formulate a mathematical model that clarifies the similarities and differences between recall and recognition mechanisms. Specifically, we formulated two alternative types of models. In one class of models, which we will call single-process models, iecall and recognition are seen as separate mechanisms. The singleprocess models assume that recognition is strictly a data-driven mechanism and recall is strictly a conceptually driven mechanism. The other class of models, which we call dual-process models, assumes that recall has one component and recognition has two components; recall involves conceptually driven retrieval, and recognition involves both data-driven and conceptually driven retrieval. The third goal of this study was to compare the $\mathrm{SP}+\mathrm{T}$ model with alternative schema-based models of memory.

\section{METHOD}

\section{Subjects}

Forty-eight students enrolled in a lower division psychology class at California State University at Fullerton participated in order to fulfill a course requirement.

\section{Materials}

The stimulus passage consisted of a tape-recorded story about a character named Jack who enacted 10 scripted activities. The Jack story was the same passage that was used in Experiment 2 of Graesser et al. (1980). There were eight experimental scripts and a buffer script at the beginning and end of the passage. The following titles summarize the content of the eight experimental scripts: "Getting Up in the Morning," "Taking the Dog to the Vet for Shots," "Washing a Car," "Cleaning an Apartment," "Eating at a Restaurant," "Visiting Someone at a Hospital," "Going to the Beach," and "Washing Clothes at a Laundromat." Before the actions of each script were presented, the script was identified by a sentence that contained the script title (e.g., "Jack decided to take his dog to the vet for shots").

The scripted activities in the Jack story were constructed systematically (see Graesser et al., 1980). Subjects in a freegeneration group provided the set of typical actions, whereas the investigators generated the set of atypical actions in each script. Typicality ratings were collected for typical and atypical actions from another normative rating group.

Each scripted activity contained 22 actions varying in typicality. Two versions of the Jack story (A and B) were prepared in order to vary the sampling of typical and atypical actions. Each version of the scripted activities contained (1) 14 typical actions presented in both Version $A$ and Version $B$, (2) 8 actions presented in Version $A$ but not in Version B, and (3) 8 actions that were presented in Version B but not in Version A. The 14 typical actions (Category 1 actions), which were common to both script versions, were not analyzed in the subsequent recall or recognition tests. There were 16 critical (Category 2 and 3 ) actions per scripted activity; these later served as test actions in the recall and recognition tasks. Half of the actions were typical and half were atypical. Since there were 8 scripted activities, Version A contained 64 actions that were not presented in Version $B$ and Version $B$ contained 64 actions that were not presented in Version $A$. Of the total 128 test actions, those with typicality ratings greater than 4.4 were considered typical and those actions with typicality ratings less than 4.4 were considered atypical. The mean of the 64 typical actions was 5.59 the mean of the atypical actions was 2.71 .

\section{Test Booklets and Counterbalancing}

Construction of the test booklets was accomplished by first assigning each experimental scripted activity a number from 1 to 8. The eight scripted activities were then divided into four pairs: $1-5,2-6,3-7,4-8$. These four pairs were assigned to four different retention intervals according to a 4 by 4 Latin square. Further counterbalancing was achieved by assigning half the subjects a recall task to one script of a pair and a recognition test to the other script of the pair; the other half of the subjects received the opposite assignment. Consequently, there were eight different groups of subjects regarding the assignment of the eight scripts to four different retention intervals and two testing formats (recall/recognition). Within these eight groups, half the subjects received Version A of the Jack story and the other half received Version $B$. 
There were three pages in the test booklet that a subject received at a particular retention interval. The first page of the booklet instructed the subject to write down all the actions that he or she recalled as having occurred during a specific scripted activity. The second page contained instructions on how to complete the recognition test (on a different scripted activity). The subjects were instructed to rate a series of actions on the following 6-point recognition scale: $1=$ action was definitely not presented in the Jack story, 2 = fairly sure action was not presented in the Jack story, $3=$ uncertain, but think action was not presented in the Jack story, $4=$ uncertain, but think action was presented in the Jack story, $5=$ fairly sure action was presented in the Jack story, and $6=$ action was definitely presented in the Jack story.

The third page of the booklet contained 16 test actions that were listed below the title of the scripted activity. There were eight test actions that were presented only in Version $A$ and eight test actions that were presented only in Version B of the Jack story. Of the eight actions in a particular version, four were typical and four were atypical.

\section{Procedure}

Subjects first listened to one version of the Jack story, which was tape-recorded at a medium rate of 175 words $/ \mathrm{min}$. Half of the subjects received Version $A$ and half received Version $B$ of the Jack story. The subjects were told to listen carefully to the tape because they would later be asked questions about the passage. Following the tape, subjects were asked to fill out a short questionnaire composed of global questions about Jack's personality. After completing this task, the subjects completed the first recall/recognition task. The test booklets were distributed according to the counterbalanced design previously described. In the recall portion of the task, the subjects were presented a script title and asked to write down all the actions they could remember from that particular script. The experimenter emphasized that the subjects should write down all actions they recalled, even if they did not seem typical of the given script. In the recognition portion, the subjects were presented a list of actions from a particular script and asked to rate each action as to whether or not it was presented in the passage, according to the 6-point recognition scale. The script title was presented at the top of the page. In the instructions, the subjects were explicitly told that some of the actions in the recognition list had not been presented.

Two days, 1 week, and 3 weeks after the initial session, the experimenter returned to the subjects' class and had them perform additional recall and recognition tasks. Following each session, the subjects were led to believe the experiment was finished. This reduced the possibility of subjects' rehearsing the script material. A given subject was never tested on a given scripted activity more than once throughout the experiment. The net result was that a given subject performed either a recall or recognition task at one of the four retention intervals for the eight scripted activities.

\section{RESULTS}

Table 1 shows the recall and recognition data for the typical and atypical actions. Hit rates and false alarm rates were computed from the recognition tests, whereas recall proportions and intrusion proportions were computed from the recall protocols. The hit and false alarm rates were computed as the proportion of "yes" responses (recognition ratings of 4,5 , or 6 ). Recall proportions and intrusion proportions were computed from the recall protocols. Correct recalls were responses that closely matched one of the eight test actions presented in a subject's version of a script. Recall intrusions were responses that closely matched one of the eight test actions in the alternate version of the same script. Responses that did not match test actions were not scored.

\section{Recall Performance}

An analysis of recall proportions showed a significant main effect for retention interval $[.287, .173, .138$, and .088 after $30 \mathrm{~min}, 2$ days, 1 week, and 3 weeks, respectively; $\min \mathrm{F}^{\prime}(3,414)=8.89, p<.01$ ], a significant main effect of typicality $[.237$ and .106 for typical and atypical actions, respectively; $\min \mathrm{F}^{\prime}(1,163)=$ $11.27, \mathrm{p}<.01]$, and a significant interaction between retention interval and typicality $\left[\min \mathrm{F}^{\prime}(3,387)=2.92\right.$, $p<.05]$. The intrusion proportions were significantly higher for typical than for atypical actions $[.153$ and .000 , respectively; $\left.\min \mathrm{F}^{\prime}(1,172)=34.56, \mathrm{p}<.01\right]$, but there was no significant main effect of retention interval $(.058, .079, .095$, and .072 for $30 \mathrm{~min}, 2$ days, 1 week, and 3 weeks, respectively) and no significant Typicality by Retention Interval interaction.

\section{Recognition Performance}

An analysis of the hit rates showed a significant main effect of retention interval $[.754, .702, .645$, and .561 after $30 \mathrm{~min}, 2$ days, 1 week, and 3 weeks, respectively; $\left.\min \mathrm{F}^{\prime}(3,289)=5.03, \mathrm{p}<.01\right]$, a significant main effect of typicality $[.733$ and .598 for typical and

Table 1

Recall and Recognition Data

\begin{tabular}{|c|c|c|c|c|c|c|c|c|}
\hline & \multicolumn{8}{|c|}{ Retention Interval } \\
\hline & \multicolumn{2}{|c|}{$30 \mathrm{Min}$} & \multicolumn{2}{|c|}{2 Days } & \multicolumn{2}{|c|}{1 Week } & \multicolumn{2}{|c|}{3 Weeks } \\
\hline & Typical & Atypical & Typical & Atypical & Typical & Atypical & Typical & Atypica \\
\hline & \multicolumn{8}{|c|}{ Recall } \\
\hline pr(recall) & .288 & .287 & .249 & .096 & .245 & .032 & .165 & .011 \\
\hline \multirow[t]{2}{*}{ pr(intrusion) } & .117 & .000 & .159 & .000 & .191 & .000 & .144 & .000 \\
\hline & \multicolumn{8}{|c|}{ Recognition } \\
\hline $\operatorname{pr}($ hit $)$ & .781 & .728 & .766 & .638 & .728 & .563 & .659 & .463 \\
\hline pr(false alarm) & .564 & .154 & .553 & .249 & .627 & .186 & .628 & .261 \\
\hline $\mathrm{d}^{\prime}$ Score & .621 & 1.619 & .613 & 1.033 & .281 & 1.029 & .081 & .543 \\
\hline
\end{tabular}

Note-From Prose Comprehension Beyond the Word by A. C. Graesser. Copyright 1981 by Springer-Verlag New York, Inc. Reprinted by permission. 
atypical actions, respectively; $\min \mathrm{F}^{\prime}(1,173)=7.14$, $\mathrm{p}<.01$ ], but no significant Retention Interval by Typicality interaction. For the false alarm rate, there was a significant main effect of typicality [.593 and .212 for typical and atypical actions, respectively; $\left.\min F^{\prime}(1,167)=70.76, p<.01\right]$, but no significant main effect of retention interval $(.359, .401, .406$, and .444 for $30 \mathrm{~min}, 2$ days, 1 week, and 3 weeks, respectively) and no significant Retention Interval by Typicality interaction.

\section{Memory Discrimination}

In addition to hit and false alarm rates, Table 1 shows $d^{\prime}$ scores for recognition performance. These $d^{\prime}$ scores provide a measure of recognition performance that corrects for guessing. Analysis of the d' scores showed that memory discrimination was uniformly higher for atypical actions than for typical actions at each retention interval. The $\mathrm{d}^{\prime}$ scores for atypical actions were significantly higher than those for typical actions $\left[1.056\right.$ and .399 , respectively; $\min F^{\prime}(1,78)=12.18$, $\mathrm{p}<.01]$. There was also a significant main effect of retention interval on $\mathrm{d}^{\prime}$ scores $[1.120, .823, .655$, and .312 for $30 \mathrm{~min}, 2$ days, 1 week, and 3 weeks, respectively; $\left.\min F^{\prime}(3,269)=6.68, p<.01\right]$. The Typicality by Retention Interval interaction was not quite significant $\left[\min F^{\prime}(3,444)=1.82, .10<p<.25\right]$.

Although these results are consistent with prior studies using the same procedures and stimulus materials, the $\mathrm{d}^{\prime}$ scores were not used in the later analyses because each data point for a subject was based on too few observations. In addition to this limitation, the $\mathrm{d}^{\prime}$ scores are applicable to recognition scores but not recall scores. Since a comparison of recall and recognition scores was desired for further analysis, it was important to compute a common measure of memory discrimination that could be applied to both recall and recognition.

\section{A Comparison of Recall and \\ Recognition Memory}

The comparison of recall and recognition memory was accomplished by computing "memory scores." The memory scores provide a common measure of memory that corrects for guessing. The false alarm rates served as an estimate of the guessing rate on the recog. nition test, whereas the intrusion proportions served as an estimate of the guessing rate for recall. Formulas 1 and 2 show how the memory scores (MS) were comuted for recall and recognition, respectively.

$\mathrm{MS}($ recall $)=$

$$
[\mathrm{p}(\text { recall })-\mathrm{p}(\text { intrusion })] /[1-\mathrm{p}(\text { intrusion })]
$$

MS $($ recognition $)=$

$$
[p(\text { hit })-p(\text { false alarm })] /[1-p(\text { false alarm })]
$$

Table 2

Obtained (O) and Predicted (P) Memory Scores for Dual Component Exponential-Linear Model

\begin{tabular}{lcccc}
\hline \multirow{2}{*}{$\begin{array}{c}\text { Retention } \\
\text { Interval }\end{array}$} & \multicolumn{2}{c}{ Typical } & & \multicolumn{2}{c}{ Atypical } \\
\cline { 2 - 3 } \cline { 5 - 5 } & $\mathrm{O}$ & $\mathrm{P}$ & $\mathrm{O}$ & $\mathrm{P}$ \\
\hline 30 Min & .194 & .201 & .287 & .299 \\
2 Days & .108 & .094 & .096 & .116 \\
1 Week & .067 & .060 & .032 & .057 \\
3 Weeks & .025 & .030 & .011 & .006 \\
& \multicolumn{4}{c}{ Recall } \\
30 Min & .498 & .510 & .677 & .655 \\
2 Days & .477 & .416 & .517 & .544 \\
1 Week & .271 & .321 & .464 & .459 \\
3 Weeks & .086 & .087 & .273 & .266 \\
\hline
\end{tabular}

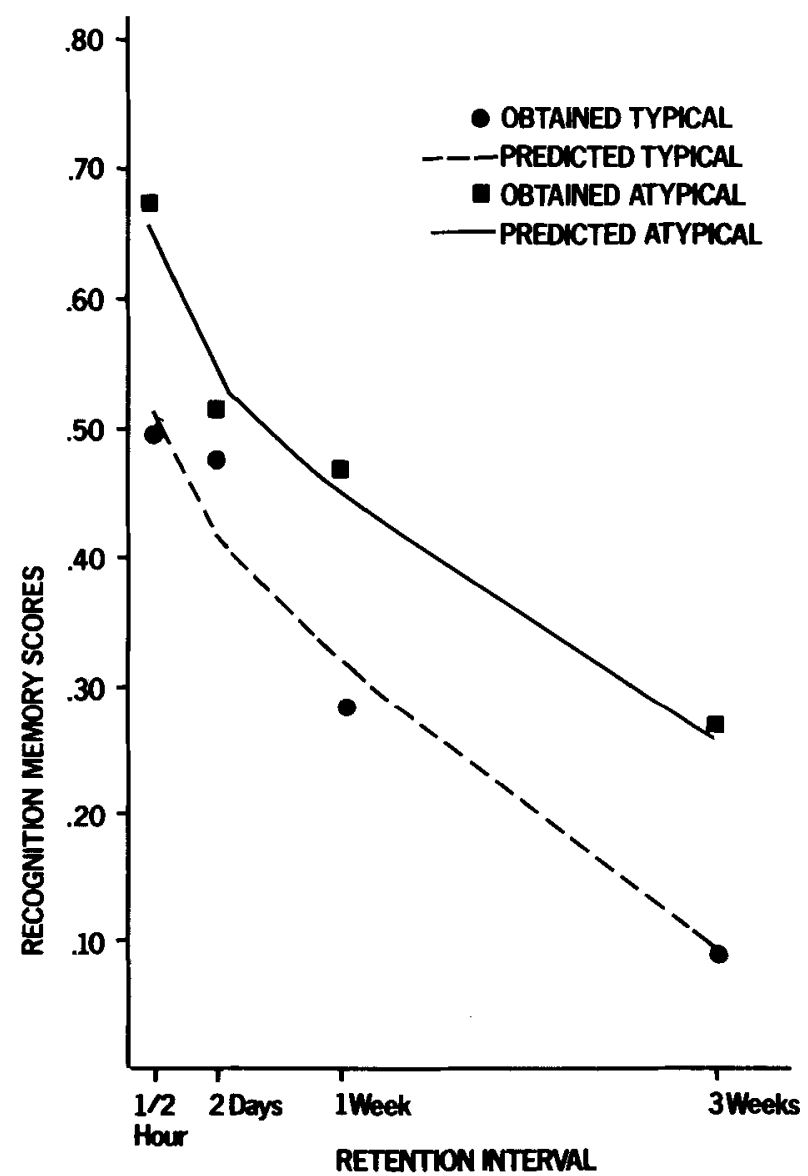
scores.

Figure 2. Obtained and predicted recognition memory

The memory scores for recognition proved to be a very good estimate of memory discrimination, since the $d^{\prime}$ scores and memory scores per action were highly correlated $(\mathrm{r}=.91, \mathrm{p}<.01)$.

Table 2 shows the memory scores for both recall and recognition, segregating typical and atypical actions at 
the four retention intervals. The memory scores were derived from the mean scores in Table 1. These scores were used in the modeling procedures that will be reported shortly. Figure 2 shows recognition memory scores for typical and atypical actions as a function of retention interval. Figure 3 shows recall memory scores for typical and atypical actions as a function of retention interval.

Figures 2 and 3 show several interesting trends. First, both recall and recognition curves are initially higher for atypical actions than for typical actions. Second, the recognition curves for atypical and typical actions maintain roughly equal slopes across the four retention intervals, whereas a crossover occurs between $30 \mathrm{~min}$ and 1 week for the recall curves. During this time the MS(recall) curve for atypical actions dips below the MS(recall) curve for typical actions. Thus, there was better recall for atypical actions after a short retention interval, but a better recall of typical actions sometime around the 2-day retention interval and thereafter. The crossover is consistent with the notion that schemata become progressively more important in guiding conceptually driven retrieval as the retention interval increases. Third, the MS(recognition) curves suggest a linear decay rate for recognition processes. Fourth, the MS(recall) curves suggest that there is an exponential decay rate for recall processes. In order to account for these trends, we will turn to some modeling procedures.

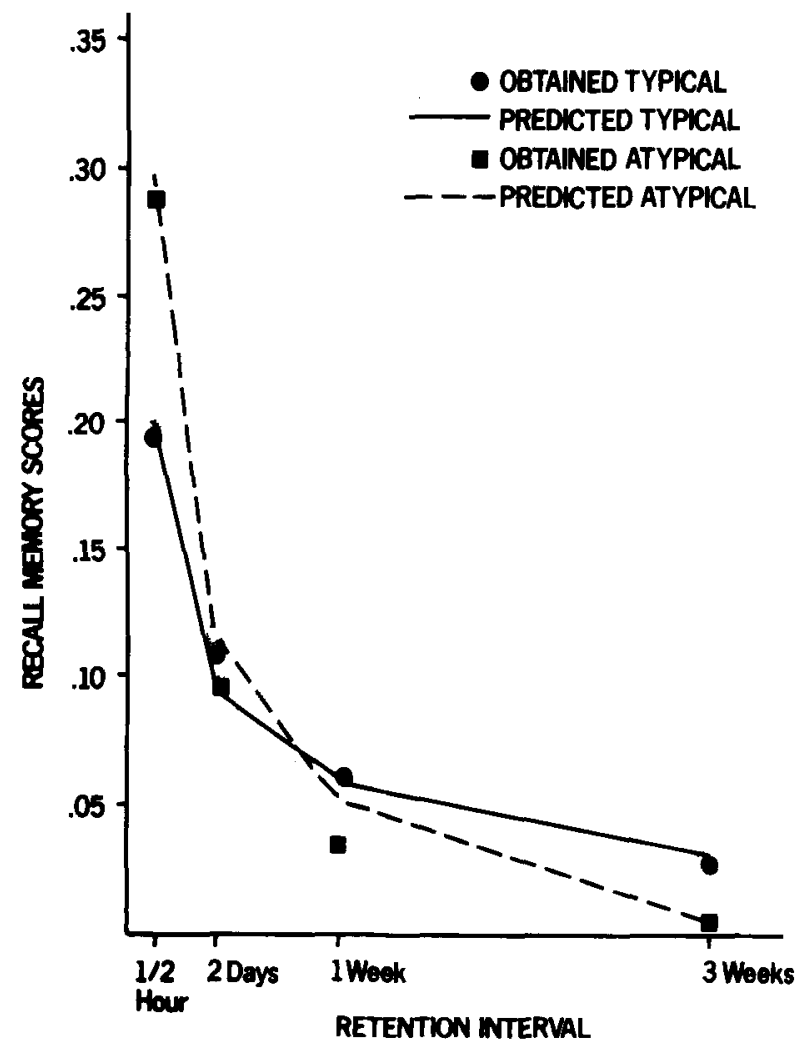

Figure 3. Obtained and predicted recall memory scores.

\section{Mathematical Modeling of the SP+T Model}

Two types of mathematical models were formulated in order to simulate the memory scores shown in Table 2. As we mentioned earlier, the distinction between the two types of models revolves around the assumptions of the recognition mechanism. The single-process model assumes a data-driven recognition mechanism; the dualprocess model assumes that recognition involves both data-driven and conceptually driven retrieval. Both single- and dual-process models assumed a conceptually driven recall mechanism. In both types of models, conceptually driven retrieval is a product of a script cue, whereas data-driven retrieval is a product of an item cue. In a recall task, the script cue is provided by the script title. In a recognition task, the script cue is provided by the script title, whereas item cues are provided by the individual test actions.

In order to assess the nature of the decay functions for recall and recognition, both the single- and dualprocess models were further specified by using the four possible combinations of linear and exponential decay functions for conceptually driven and data-driven retrieval mechanisms. This resulted in eight models, four single-process models and four dual-process models. Table 3 shows the mathematical formulas for the eight models that were tested. Each model includes three types of parameters. The intercept corresponds to the initial level of acquisition, the slope specifies a forgetting rate, and the retention parameter is the time interval.

For the recall retention curve in a given single-process model, the slope is designated as the letter $b$ and the intercept as the letter a. In the recognition curve, the slope is designated as the letter $\mathrm{d}$ and the intercept as the letter $\mathrm{c}$. The time parameter is always designated as the letter $\mathrm{t}$. Days were used as the unit of time in order to show reasonably large values for the slope parameter.

For the dual-process models, we had parameters and letter designations similar to those for the single-process models. The only mathematical difference between the two types of models is the formulation of the MS(recognition) equations. The dual-process models require a more complex MS(recognition) equation in order to capture both conceptually driven and data-driven processes. The MS(recognition) formulas are best described by breaking them into two parts. The first part of the formula, which is the same as the corresponding MS(recall) formula, reflects the probability of accessing an item through conceptually driven retrieval. The second part of the formula reflects the probability of accessing an item through data-driven retrieval. Data-driven and conceptually driven retrieval were assumed to be stochastically independent.

Computer programs were written to estimate best-fit parameters for each of the eight models. Least squares values were used as the criterion to assess the goodness of fit between the obtained and predicted memory scores. All of the models had the same number of parameters.

Modeling results. Table 4 shows the best-fit values of 
Table 3

Equations for the Eight Models

\begin{tabular}{|c|c|c|}
\hline & Single-Process Models & Dual Process Models \\
\hline Linear-Linear & $\begin{array}{l}\text { MS(recall) }=a+b t \\
M S(\text { recognition })=c+d t\end{array}$ & $\begin{array}{l}\text { MS(recall) }=a+b t \\
\text { MS (recognition) }=(a+b t)+(1-a-b t) *(c+d t)\end{array}$ \\
\hline Linear-Exponential & $\begin{array}{l}\text { MS(recall) }=a+b t \\
\text { MS(recognition) }=c+d \log t\end{array}$ & $\begin{array}{l}\text { MS }(\text { recall })=a+b t \\
\text { MS }(\text { recognition })=(a+b t)+(1-a-b t) *(c+d \log t)\end{array}$ \\
\hline Exponential-Linear & $\begin{array}{l}\text { MS(recall) }=a+b \log t \\
\text { MS(recognition) }=c+d t\end{array}$ & $\begin{array}{l}\text { MS }(\text { recall })=a+b \log t \\
\text { MS }(\text { recognition })=(a+b \log t)+(1-a-b \log t) *(c+d t)\end{array}$ \\
\hline Exponential-Exponential & $\begin{array}{l}\text { MS }(\text { recall })=a+b \log t \\
\text { MS(recognition) }=c+d \log t\end{array}$ & $\begin{array}{l}\text { MS(recall) }=a+b \log t \\
\text { MS (recognition) }=(a+b \log t)+(1-a-b \log t) *(c+d \log t)\end{array}$ \\
\hline
\end{tabular}

Table 4

Parameter Values and Least Squares for the Eight Models

\begin{tabular}{|c|c|c|c|c|c|c|c|c|c|}
\hline & \multicolumn{8}{|c|}{ Parameter } & \multirow{3}{*}{$\begin{array}{l}\text { Least } \\
\text { Square }\end{array}$} \\
\hline & \multicolumn{4}{|c|}{ Typical } & \multicolumn{4}{|c|}{ Atypical } & \\
\hline & $\mathrm{a}$ & $\mathrm{b}$ & $c$ & $\mathrm{~d}$ & a & $\mathrm{b}$ & c & $\mathrm{d}$ & \\
\hline & \multicolumn{9}{|c|}{ Single-Process Models (Recall-Recognition) } \\
\hline Linear-Linear & .129 & -.004 & .480 & -.019 & .133 & -.008 & .602 & -.016 & .051 \\
\hline Exponential-Linear & .117 & -.027 & .480 & -.019 & .137 & -.047 & .602 & -.016 & .017 \\
\hline Linear-Exponential & .129 & -.004 & .367 & -.059 & .133 & -.008 & .519 & -.059 & .082 \\
\hline \multirow[t]{2}{*}{ Exponential-Exponential } & .117 & -.027 & .367 & -.059 & .137 & -.047 & .519 & -.059 & .048 \\
\hline & \multicolumn{9}{|c|}{ Dual Process Models (Conceptually Driven/Data Driven) } \\
\hline Linear-Linear & .148 & -.008 & .395 & -.016 & .207 & -.012 & .469 & -.008 & .051 \\
\hline Exponential-Linear & .113 & -.027 & .387 & -.016 & .148 & -.047 & .508 & -.012 & .009 \\
\hline Linear-Exponential & .117 & -.008 & .324 & -.047 & .133 & -.008 & .477 & -.051 & .059 \\
\hline Exponential-Exponential & .117 & -.027 & .297 & -.047 & .137 & -.047 & .457 & -.039 & .042 \\
\hline
\end{tabular}

the parameters of each model. In addition, Table 4 includes the least squares value for each of the eight models. The least squares ranged from .009 for the dual-process exponential-linear model to .082 for the single-process linear-exponential model. In all cases, the least square values for the single-process models exceeded or equaled the corresponding (with analogous decay functions) least square values for the dual-process models. The results of this analysis clearly indicate that the dual-process exponential-linear model most closely simulated the obtained data. Based on these results, we rejected all but the dual-process exponential-linear model. The fact that this model best simulated the obtained data supports the SP+T model's conceptually driven recall mechanism and conceptually driven plus data-driven recognition mechanisms.

Figures 2 and 3 plot the obtained MS(recall) and MS(recognition) values for both typical and atypical actions at the four retention intervals. The lines in these figures correspond to expected values predicted by the dual-process exponential-linear model. Inspection of these figures reveals how closely the SP+T model simulates the actual data.

The present formulation of the SP+T model is an allor-none model. In the past, all-or-none models have shown shortcomings when predicting recognition memory of word lists. In our case, however, an all-or-none (high-threshold) model was apparently adequate for modeling scripted passages. It would be possible to translate our current mathematical formulation into a more complex model that is derived from signal detection theory (Kintsch, 1977), but for the present we adopted the modeling procedures as previously described.

The modeling procedures were also performed on an alternate set of memory scores that utilized a different correction for guessing. These scores were simply difference scores, $p($ recall $)-p($ intrusion $)$ and $p($ hit $)-p($ false alarm). The results of the modeling were virtually identical to those with the memory scores in Tables 2 and 4 .

\section{DISCUSSION}

In light of the results obtained in this study, we will reevaluate the $\mathrm{SP}+\mathrm{T}$ model, focusing on the following four issues: typicality, retrieval task, retention interval, and guessing.

\section{Typicality}

Our results indicate that it is inappropriate to make general statements about memory for typical vs. atypical actions without considering the retention interval and the type of retrieval task. The retention curves for typical and atypical actions showed substantially different patterns when considering recall vs. recognition tasks. However, the data from the 30 -min retention interval clearly indicates that atypical actions are retrieved better than typical actions in both recall and recognition tasks. 
The SP+T model assumes that the "typicality effect" is a product of differences in encoding for typical vs. atypical information. The model asserts that the encoded representation of a passage contains three sets of actions: (1) typical actions that appeared in the text, (2) typical actions that were inferred, and (3) atypical actions that were presented in the text. When an individual attempts to retrieve a typical action from the memory representation, it is difficult to discriminate between the stated and inferred actions. On the other hand, the atypical actions are represented in a distinctive way and easily accessed by virtue of the tags. In both recall and recognition tasks, individuals are able to retrieve a high proportion of the typical and atypical test actions. In addition, however, the data also show high intrusion and false alarm rates for typical actions. When guessing is partialed out, memory scores are highest for atypical information in both recall and recognition tasks.

\section{Retrieval Task}

The second issue addresses differences between recall and recognition retrieval mechanisms. The most dramatic finding involves the different trends that occur across all retention intervals. The results of the modeling showed that the dual-process exponential-linear mathematical formulation of the SP+T model was most accurate in simulating the obtained data. In fact, this formulation was twice as accurate as the next best formulation, when using least squares as a measure of goodness of fit. The overall trend of the data was simulated very accurately when conceptually driven retrieval was captured by an exponential decreasing function over time and data-driven retrieval was captured by a linear decreasing function. The proposed $\mathrm{SP}+\mathrm{T}$ model asserts that the recall of information is directed by conceptually driven retrieval, whereas recognition is the product of both conceptually driven and data-driven retrieval mechanisms. In both recall and recognition tasks, an individual was presented with a script title that guides the conceptually driven retrieval mechanism. In recognition, the individual also receives a test item that directs the data-driven retrieval process.

The exponential decay function for conceptually driven retrieval is consistent with most theories of forgetting that have been postulated since the era of Ebbinghaus (see Kintsch, 1977). However, the linear function, which best describes data-driven retrieval, is not common in memory theories. Most studies that plot the forgetting rate for recognition memory have focused on short-term memory, in which decay is very rapid. Our results show that the rate of forgetting is much more gradual and constant at longer retention intervals. This gradual loss of item retrievability is perhaps due to the multiple dimensions that are encoded and retrieved in data-driven retrieval. The memory trace for each item is encoded along several dimensions, and these same dimensions are reinstated during data-driven retrieval.
The decay rate for one dimension is most likely exponential. However, since data-driven retrieval can be accomplished through any subset of the dimensions, the net forgetting rate is very much reduced. While the actual rate of forgetting may be exponential, it is so slight a decrease that a linear function provides a very good fit.

\section{Retention Interval}

The third issue of importance involves the retrieval of information over time. Our results clearly indicated that the probability of recalling a test item via conceptually driven retrieval exponentially decreases over time. However, the data-driven component of the recognition curves is best fit to a linear function. In addition, there is a crossover in the typical and atypical recall curves between the 30-min and 3-week retention intervals. The overall pattern of recall scores is consistent with the SP+T model's assumption that the generic schema becomes progressively more important in guiding conceptually driven retrieval over time. At short retention intervals, memory is primarily reproductive, with most of the information being retrieved from the memory trace. As the retention interval increases, the tagged atypical actions in the trace become less accessible because retrieval becomes more dependent upon the generic schema. As retrieval becomes more dependent upon the generic schema, memory becomes more reconstructive.

The $\mathrm{SP}+\mathrm{T}$ model assumes that recognition involves both conceptually driven and data-driven retrieval processes. In order to estimate the likelihood of accessing an item through data-driven retrieval, we needed to partial out (1) access to the item through conceptually driven retrieval and (2) guessing. Both these components could be estimated. Conceptually driven retrieval was derived for the recall data and guessing in recognition tests was estimated from the false alarm rate. Once these components were partialed out, the probability of accessing an item through data-driven retrieval showed a trend much different from that shown by conceptually driven retrieval. While conceptually driven retrieval was best simulated through an exponential function, datadriven retrieval followed a linear decay rate. In addition, while conceptually driven retrieval showed differential rates of decay for typical and atypical actions, the slopes of data-driven retrieval actions were roughly equal for typical and atypical actions. In fact, the slope for atypical actions was slightly lower (less steep) than that for typical actions (see Table 4).

\section{Guessing}

We have already described both the rationale and the methods for our correction for guessing. The SP+T model assumes that guessing is primarily a result of encoding processes as opposed to retrieval processes (see Graesser, 1981). This assumption has relevance to 
the representational assumptions of the $\mathrm{SP}+\mathrm{T}$ model. The SP+T model assumes that the memory trace contains both stated and inferred typical actions, as well as stated atypical actions. We have reported that the guessing rate for atypical actions is very slight, whereas the guessing rate for typical actions is relatively high in both recall and recognition tasks.

\section{Alternative Models}

How do the predictions of other schema-based models compare with the predictions of the SP+T model? We will now consider some alternative models: (1) the partial copy model of Bower et al. (1979), (2) the depth-of-processing models (e.g., Hastie, 1980), and (3) the filtering models (e.g., Cantor \& Mischel, 1979; Taylor \& Crocker, 1981). Since the goals under which these different models were developed are somewhat different from the goals of the SP+T model, it is not always possible to assess directly the other models' predictions. When possible, however, predictions will be made based upon the available articulation of the different models. It is our contention that the four issues introduced previously should be addressed in a complete schema model.

Bower et al. (1979) developed the partial copy model to describe how script-based test is represented and retrieved from memory. The partial copy model assumes that when a script-based text is read, the explicitly stated actions are recorded in an episodic memory trace. In addition, information in the generic script becomes activated in the form of occurrence tags (not to be confused with the tags in the SP+T model). Explicitly stated typical actions receive two tags (high activation), whereas a single tag is formed for inferred typical actions (low activation). The partial copy model assumes that forgetting occurs in two ways. First, the episodic memory trace, which contains all explicitly stated actions, decays rather quickly at an exponential rate. Second, the occurrence tags in the generic script decay independently of the episodic memory trace. Through the loss of the episodic memory trace, the individual forgets exactly what was contained in the text. Even after the loss of the episodic trace, however, the individual can rely upon the tagged actions in the generic script to construct an approximation of the original text. Eventually, the occurrence tags decay from the generic script and the individual is unable to retrieve what was explicitly stated in the text.

The partial copy model assumes a three-stage recognition process. First, the episodic memory trace is searched for a particular item. If a match occurs, the subject will make a positive recognition judgment. If no match occurs at this point, the search goes to the action in the generic script that corresponds to the test item. If the level of activation of the action in the generic script is below some threshold, then the item is judged as having not been presented. If the threshold is exceeded, then the item is judged as having been presented.
The partial copy model makes specific predictions as to the fate of irrelevant (atypical) information that occurs in script-based text. At short retention intervals, irrelevant information is retrieved by virtue of the episodic memory trace. Over a period of time, however, the loss of the episodic trace results in the loss of the irrelevant information. This prediction was not supported by the present findings. After a long retention interval, when the episodic trace was completely decayed, there was still memory for atypical actions.

Models based on the depth-of-processing notion have also been proposed to account for the representation and retrieval of schema-based person information. Hastie (1980) outlined such a model, which states that information regarding a stereotype is represented in memory as a propositional network. Items in the network are stored as nodes that are linked together. The strength of the links is a function of active memory processing at input. Items that are unusual or seen as important receive extra processing time and elaboration (Craik \& Lockhart, 1972). Forgetting is the product of links' becoming less accessible due to changes in the propositional network. These changes in the propositional network are a product of more recent information entering memory.

A model based on depth of processing can account for the typicality effect by assuming that atypical actions are processed more deeply at the time of input. This explanation, however, does not seem probable, since Graesser et al. (1979) found that variations in available processing resources had no effect on the memory of typical and atypical actions. In addition, a model based upon the assumption of deeper processing for atypical actions could not account for the crossover in the recall curves for typical and atypical actions between 30-min and 2-day retention intervals (see also Graesser et al., 1980). If atypical actions were processed more deeply, they would be remembered better regardless of the length of the retention interval.

Another type of schema model has been developed by researchers in the area of social cognition. This model views schemata as templates that select the most useful information from the environment and then set up guidelines for processing (Cantor \& Mischel, 1979; Crocker \& Taylor, 1981). We have chosen the term "filtering model" to refer to this type of model because the models assume that schemata selectively filter out "irrelevant" (atypical) information. Any atypical information that was not "filtered out" would be loosely associated with the cohesively related typical information. Individuals would therefore find it difficult to retrieve the remotely associated atypical information from memory. A filtering model, as such, has not been specifically defined, but much of the social cognition literature centers around these implicit assumptions.

The research that is supportive of a filtering model is based on one critical observation. Most of the social cognition research reports better memory for typical 
than for atypical information. Unfortunately, these researchers have failed to correct for guessing when analyzing the data. It is our contention that if guessing had been accounted for in the research supporting the filtering model, the results would reflect better memory for atypical than for typical information.

This study was conducted with three goals in mind. First, we assessed how well the SP+T model accounts for the effects of typicality, retention interval, and retrieval task on memory for scripted activities. Second, we provided a mathematical formulation of the SP+T model. Third, we compared the SP+T model with some alternative schema-based memory models. We feel that the results of this study demonstrate the ability of the $\mathrm{SP}+\mathrm{T}$ model and the mathematical formulation of the $\mathrm{SP}+\mathrm{T}$ model to explain and simulate memory for scripted activities. We are not offering the current formulation of the SP+T model as a final explanation of memory for scripted activities. We do feel, however, that the SP+T model provides a good fit to the data and a fruitful direction for future research.

\section{REFERENCES}

Atkinson, R. C., \& Juola, J. F. Search and decision processes in recognition memory. In D. H. Krantz, R. C. Anderson, \& P. Suppes (Eds.), Contemporary developments in mathematical psychology. San Francisco: Freeman, 1974.

Bartuett, F. C. Remembering. Cambridge, Mass: Cambridge University Press, 1932.

Bower, B. H., Black, J. B., \& Tunner, T. J. Scripts in memory for text. Cognitive Psychology, 1979, 11, 177-220.

Brown, J. Recall and recognition. New York: Wiley, 1976.

CAntor, N., \& Mischel, W. Prototypicality and personality: Effects on free recall and personality impression. Journal of Research in Personality, 1979, 13, 187-203.

Cohen, C. E. Person categories and social perception: Testing some boundaries of the processing effects of prior knowledge, Journal of Personality and Social Psychology, 1981, 40, 441-452.

Craik, F. I. M., \& LockharT, R. S. Levels of processing: A framework for memory research. Journal of Verbal Learning and Verbal Behavior, 1972, 11, 671-684.

D'Andrade, R. G. Memory and the assessment of behavior. In $H$. Blalock (Ed.), Measurement in the social sciences. Chicago: Aldine-Atherton, 1974.

Graesser, A. C. Prose comprehension beyond the word. New York: Springer-Verlag, 1981.

Graesser, A. C., Gordon, S. E., \& SAwYer, J. D. Recognition memory for typical and atypical actions in scripted activities: Tests of a script pointer + tag hypothesis. Journal of Verbal Learning and Verbal Behavior, 1979, 18, 319-332.

Graesser, A. C., Woll, S. B., Kowalski, D. J., \& Smith, D. A. Memory for typical and atypical actions in scripted activities. Journal of Experimental Psychology: Human Learning and Memory, 1980, 6, 503-515.
HAstie, R. Memory for behavioral information that confirms or contradicts a personality impression. In R. Hastie, T. Ostrom, E. Ebbesen, R. S. Wyer, D. L. Hamilton, D. Carlston (Eds.), Person memory. Hillsdale, N.J: Erlbaum, 1980.

Kint8ch, W. Memory and cognition. New York: Wiley, 1977.

Kintsch, W., \& van Dijk, T. A. Toward a model of text comprehension and production. Psychological Review, 1978, 85, 363-394.

Mandler, G. Organization and recognition. In E. Tulving \& W. Donaldson (Eds.), Organization and memory. New York: Academic Press, 1972.

Mandler, G. Recognizing: The judgment of previous occurrence. Psychological Review, 1980, 87, 252-271.

Mandler, G., \& Boeck, W. J. Retrieval processes in recognition. Memory \& Cognition, 1974, 2, 613-615.

Mandle R, J. M. Categorical and schematic organization in memory. In C. R. Puff (Ed.), Memory organization and structure. New York: Academic Press, 1979.

Minsky, M. A. A framework for representing knowledge. In P. H. Winston (Ed.), The psychology of computer vision. New York: McGraw-Hill, 1975.

Norman, D. A., \& Bosnow, D. G. Descriptions: An intermediate stage in memory retrieval. Cognitive Psychology, 1979, 11, $107-123$.

Rabinowitz, J. C., Mandler, G., \& Patterson, K. E. Determinants of recognition and recall: Accessibility and generation. Journal of Experimental Psychology: General, 1977, 106, 302-329.

Rumelhart, D. E., \& Ortony, A. The representation of knowledge in memory. In R. C. Anderson, J. J. Spiro, \& W. E. Montague (Eds.), Schooling and the acquisition of knowledge. Hillsdale, N. J: Erlbaum, 1977.

Schank, R. C., \& Abelson, R. Scripts, plans, goals, and understanding. Hillsdale, N.J: Erlbaum, 1977.

SpIRo, R. J. Remembering information from text: The "state of the schema" approach. In R. C. Anderson, R. J. Spiro, \& W. C. Montague (Eds.), Schooling and the acquisition of knowledge. Hillsdale, N. J: Erlbaum, 1977.

TAYlor, S. E., \& Crocker, J. Schematic bases of social information processing. In E. T. Higgins, C. P. Herman, \& M. P. Zanna (Eds.), Social cognition: The Ontario symposium on personality and social psychology. Hillsdale, N.J: Erlbaum, 1981.

Thorndye, P. W., \& Hayes-Roth, B. The use of schemata in the acquisition and transfer of knowledge. Cognitive Psychology, 1979, 11, 82-106.

Tulving, E., \& Thomson, D. M. Encoding specificity and retrieval processes in episodic memory. Psychological Review, $1973,80,352-373$.

Woodworth, R. S. Dynamics of behavior. New York: Holt, 1958.

Woodworth, R. S., \& Schlosberg, H. Experimental Psychology. New York: Holt, Rinehart, \& Winston, 1954.

WYer, R. S., \& SRULl, T. K. The processing of social stimulus information: A conceptual integration. In R. Hastie, T. Ostrom, E. Ebbesen, R. S. Wyer, D. L. Hamilton, \& D. Carlston (Eds.), Person memory. Hillsdale, N.J: Erlbaum, 1980.

(Received for publication December 17, 1980; revision accepted June 30,1981 .) 